\title{
PERFIL NUTRICIONAL EM CRIANÇAS DE 2 A 5 ANOS NO MUNICÍPIO DE SERRO, MINAS GERAIS
}

\author{
Aline M. VARGAS ${ }^{1}$ \\ Ana Carolina ABREU ${ }^{1}$ \\ Bruno L. MACHADO ${ }^{1}$ \\ Ellen C. A. RODRIGUES ${ }^{1}$ \\ Natália N. NEVES ${ }^{1}$ \\ Mariela Dutra Gontijo MOURA ${ }^{2}$ \\ Frederico V. MONKEN ${ }^{3}$ \\ Soraya Mattos Camargo GROSSMANN ${ }^{4 *}$
}

1Acadêmicos, Universidade Vale do Rio Verde - UNINCOR, Belo Horizonte- MG.

${ }_{2}$ Cirurgiã-dentista, Doutora, Faculdade de Odontologia, Universidade Vale do Rio Verde - UNINCOR, Belo HorizonteMG.

3Médico do município do Serro- MG.

4Cirurgiã-dentista, Pós doutora, Faculdade de Odontologia, Universidade Vale do Rio Verde - UNINCOR, Belo

Horizonte- MG.

*Correspondência para/Correspondence to:

Dra. Soraya de Mattos Camargo Grossmann

Universidade Vale do Rio Verde- UNINCOR

Campus Belo Horizonte

Avenida Amazonas, 1700

Barro Preto 30180-001

Belo Horizonte-Minas Gerais

Brasil

E-mail: prof.soraya.grossmann@unincor.edu.br

Telephone: 55 (31) 3344-1366

Recebido em: 02/12/2014 - Aprovado em: 03/06/2015 - Disponibilizado em: 15/07/2015

\section{RESUMO}

Objetivo: O trabalho analisa o perfil nutricional de crianças entre 2 e 5 anos, caracterizando dados antropométricos peso e idade.

Métodos: Foi estudada uma amostra de 722 crianças, de ambos os sexos, pertencentes à famílias cadastradas no Programa de Saúde da Família, Brasil. Os percentis de peso por idade foram calculados e categorizados em grupos, de acordo com a classificação do SISVAN.

Resultados: A prevalência de crianças nutridas foi de $86,14 \%$, no entanto, a desnutrição foi de10,66\%, valor muito elevado quando comparado ao do Brasil de 2,12\%. Provavelmente, esse resultado é devido ao fato da cidade estar localizada numa das regiões mais pobres do Brasil. Além disso, o estudo mostrou um índice local de sobrepeso de crianças de 5 anos mais alto quando comparado às demais idades. 
Conclusão: Portanto, essas crianças desnutridas e com sobrepeso merecem maior atenção por parte das autoridades locais, através de políticas públicas e programas de promoção da saúde com foco diferenciado, visando índices mais positivos ou, no mínimo, similares ao restante do Brasil.

Palavras-chave: Antropometria, Estado Nutricional, Desnutrição Proteico-Calórica, Pré-escolar, Prevalência.

\begin{abstract}
Objective: The paper analyzes the nutritional status of children between 2 and 5 years, featuring anthropometric data for weight and age.

Methods: A sample of the study was 722 children of both sexes, belonging to families enrolled in Family Health Program living in Brazil. The weight percentiles for each age were calculated and categorized into groups according to the classification of SISVAN.

Results: The prevalence of nourished children was $86.14 \%$, however, for malnourished children was $10.66 \%$, which is very high when it is compared to Brazil, which is about $2.12 \%$. Probably, this result is due to the city's location in one of the poorest regions of Brazil. Furthermore, the study showed a local index of overweight in 5 year old children highest when compared to lower ages.

Conclusion: Therefore, these malnourished and overweight children deserve greater attention from local authorities. Acting through public policy and health promotion programs with different focus, authorities should aim indices more positive or at least similar to the rest of Brazil.
\end{abstract}

Key words: Anthropometry, Nutritional Status, Protein-Energy Malnutrition, Preschool, Pevalence. 


\section{INTRODUÇÃO}

O diagnóstico das condições nutricionais das crianças constituem forma prática de avaliar as tendências da saúde infantil e o grau com que vêm sendo atendidos os direitos humanos elementares da população infantil (STRUFALDI et al., 2003). O estado nutricional infantil é um indicador de saúde global e permite monitorar o crescimento e detectar possíveis agravos à saúde e riscos nutricionais. A ampliação da observação de indicadores sociais e biológicos de crianças uma determinada comunidade por instituições de ensino e pesquisa próximas geograficamente possibilita o planejamento de estratégias de intervenção mais eficazes e eficientes no âmbito das realidades regionais (FERREIRAMARIM; FABBRO, 2012; SPERANDIO et al., 2011; SILVA et al., 2008).

Apesar do declínio acelerado na última década, a desnutrição continua sendo um problema de grande relevância para as crianças menores de cinco anos e o excesso de peso e a obesidade estão frequentemente presentes nas crianças maiores de cinco anos em todos os grupos de renda e em todas as regiões brasileiras (BRASIL, 2009). O comprometimento nutricional está associado à maior incidência e gravidade de doenças infecciosas, elevação nas taxas de mortalidade na infância, retardo no desenvolvimento psicomotor, dificuldades escolares, diminuição da estatura e da capacidade reprodutiva na idade adulta (FERREIRAMARIM; FABBRO, 2012; SPERANDIO et al., 2011; MONTEIRO; CONDE, 2000; BARROSO; SICHIERI; COSTA, 2008).

A antropometria tem sido amplamente utilizada com sucesso na avaliação da saúde e de riscos nutricionais em crianças, auxiliando na definição de prioridades no planejamento, implementação e avaliação de programas (WORLD HEALTH ORGANIZATION, 1995). Na faixa etária entre dois a cinco anos, o grau de nutrição avaliado a partir dos dados antropométricos peso por idade, que é é a relação entre a massa corporal e a idade cronológica da criança, representa os graus de carência aos quais as crianças estão submetidas, apesar de não estabelecer o processo específico leva às crianças à desnutrição. O perfil nutricional das crianças é uma informação valiosa para o planejamento de ações, visando a prevenção e a programação de cuidados dirigidos às crianças com déficits já instalados e implementando ações que possibilitem a prevenção de seus efeitos (BRASIL, 2008; TUMA; COSTA; SCHMITZ, 2005; SANTOS; LEAO, 2008; LEON-VALENCIA; TERRY-BERRO; QUINTANA-JARDINES, 2009). Assim, o presente estudo tem por 
objetivo analisar o perfil nutricional das crianças entre 2 e 5 anos no município de Serro, Minas Gerais, a partir de medidas antropométricas.

\section{METODOLOGIA}

Por meio deste estudo transversal, foram incluídas todas as crianças de dois a cinco anos, de ambos os sexos, durante o período de julho a dezembro de 2010, pertencentes à famílias cadastradas no Programa de Saúde da Família (PSF) do município de Serro, Minas Gerais, Brasil. Os agentes comunitários de saúde (ACS) agruparam as crianças de acordo com os sete PSF onde foram atendidas. A partir dos dados arquivados pela Secretaria Municipal de Saúde de Serro, foram calculados os percentis de peso por idade de todas as crianças pesadas pelos ACS.

Em seguida, as crianças foram categorizadas em grupos: Sobrepeso (SP), Nutrido (N), Baixo Peso (BP) e Muito Baixo Peso (MBP) para comparar e avaliar o crescimento da criança a valores definidos na escala padrão. Esta escala analisou o percentil da criança de acordo com o peso e a idade da mesma, lançados no gráfico. As tabelas de medições foram categorizadas como MBP percentil $<0,1 ; \mathrm{BP}->$ ou $=0,1$ e $<3$; $\mathrm{N}>$ ou $=3$ e $<$ ou $=97 ;$ SP $>97$, de acordo com o
SISVAN (BRASIL, 2008). Crianças com BP ou MBP foram consideradas desnutridas, segundo os critérios do SISVAN (BRASIL, 2008). Também foi realizada a comparação do perfil nutricional das crianças dos PSF dessa cidade com os níveis nacionais para posterior apresentação aos gestores do município.

Esse projeto foi aprovado pela Secretaria Municipal de Saúde de Serro Minas Gerais, sendo submetido e aprovado posteriormente ao Comitê de Ética e Pesquisa da Universidade Vale do Rio Verde (UNINCOR). Todos os dados coletados foram armazenados em um banco de dados, seguido da realização da análise estatística.

\section{RESULTADOS}

Dentre as 722 crianças avaliadas neste estudo, 190 (26,31\%) tinham dois anos, 224 $(31,02 \%)$ três anos, $248(34,34 \%)$ quatro anos, $60(8,31 \%)$ cinco anos de idade.

A prevalência de crianças nutridas foi $86,14 \%, 3,18 \%$ para crianças com SP; $8,17 \%$ para crianças com BP e 2,49\% para crianças com MBP. A prevalência de desnutrição foi 10,66\%. Avaliando-se o estado nutricional das crianças separadamente por sexo, observou-se que crianças com três anos apresentaram os menores índices de SP $(2,23 \%)$ e os melhores índices de $\mathrm{N}(87,50 \%)$. Os resultados referentes a todas as crianças avaliadas de 
acordo com o SISVAN em Peso/Idade estão sumarizadas no gráfico 1 e na tabela 1 .
Gráfico 1: Classificação nutricional das crianças envolvidas no estudo

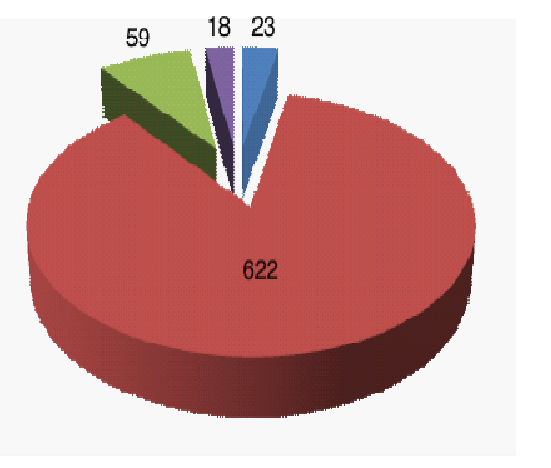

Legenda: SP (Sobrepeso); N (Nutrido); BP (Baixo peso); MBP (Muito Baixo Peso).

Fonte: Autores

Tabela 1: Análise nutricional geral das crianças avaliadas na cidade do Serro-MG (2011)

\begin{tabular}{|l|c|c|c|c|c|c|c|c|c|c}
\hline & \multicolumn{2}{|c|}{ SP } & \multicolumn{2}{c|}{$\mathrm{N}$} & \multicolumn{2}{c|}{ BP } & \multicolumn{3}{c}{ MBP } & \multicolumn{2}{c}{ TOTAL } \\
\hline Idade & $\mathrm{n}^{\text {o }}$ & $(\%)$ & $\mathrm{n}^{\text {o }}$ & $(\%)$ & $\mathrm{n}^{\text {o }}$ & $(\%)$ & $\mathrm{n}^{\text {o }}$ & $(\%)$ & $\mathrm{n}^{\text {o }}$ & $(\%)$ \\
\hline 2 anos & 6 & 3,15 & 162 & 82,26 & 15 & 7,89 & 7 & 3,68 & 190 & 26,31 \\
\hline 3 anos & 5 & 2,23 & 196 & 87,50 & 20 & 8,92 & 3 & 1,33 & 224 & 31,02 \\
\hline 4 anos & 9 & 3,62 & 212 & 85,48 & 20 & 8,06 & 7 & 2,89 & 248 & 34,34 \\
\hline 5 anos & 3 & 5,00 & 52 & 86,66 & 3 & 5,00 & 2 & 3,33 & 60 & 8,31 \\
\hline Total & 23 & 3,18 & 622 & 86,42 & 58 & 8,17 & 19 & 2,63 & 722 & 100 \\
\hline
\end{tabular}

Legenda: SP (Sobrepeso); N (Nutrido); BP (Baixo peso); MPB (Muito Baixo Peso).

Fonte: Autores 
No entanto, quando foi comparado os grupos etários em relação a categoria nutricional observou-se uma maior proporção de crianças de SP na faixa etária de cinco anos e maior MBP na faixa etária de dois anos, conforme observado no gráfico 2 .

Gráfico 2: Categoria nutricional das crianças por idade

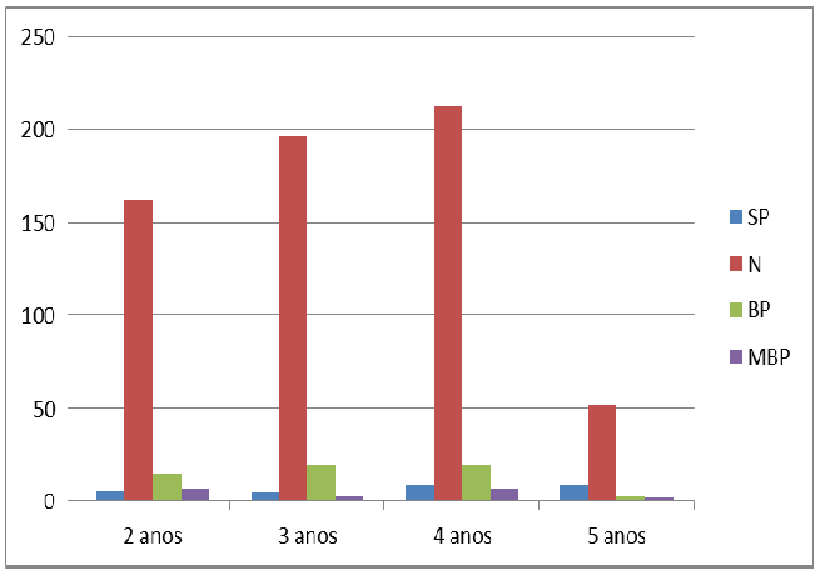

Legenda: SP (Sobrepeso); N (Nutrido); BP (Baixo peso); MPB (Muito Baixo Peso).

Fonte: Autores

\section{DISCUSSÃO}

A população de 21686 habitantes do Serro, Minas Gerais, caracteriza-se por grande precariedade socioeconômica. A proporção de menores de 5 anos na população do Serro é $11,82 \%$, superior quando comparado ao estado de Minas Gerais e ao Brasil que são, respectivamente, $9,04 \% \mathrm{e}$ 9,67\% (BRASIL, 2004). Na última década, tanto em âmbito nacional quanto regional, observa-se um declínio marcante na prevalência da desnutrição em crianças menores de cinco anos, no entanto, aumentase a prevalência de sobrepeso e obesidade na população brasileira. Estabelece-se, dessa forma, um antagonismo de tendências temporais entre desnutrição e obesidade, definindo uma das características marcantes do processo de transição nutricional do país (FILHO; RISSIN, 2003). É interessante ressaltar que o presente estudo é particularmente útil para o estabelecimento de prioridades, condutas e definição de estratégias na Atenção Básica à Saúde, situando o perfil nutricional das crianças entre dois e cinco anos na realidade regional de Serro, Minas Gerais.

Diversas formas de mensurar estados de nutrição, BP e SP podem ser utilizadas, no entanto, o presente estudo levou em consideração o percentil da criança de acordo com o peso e a idade da mesma, semelhante ao estudo de Santos (SANTOS; LEAO, 2008). Considerando-se a classificação do SISVAN, observou-se que a prevalência de crianças nutridas entre as crianças estudadas foi de $86,14 \%$; sendo que $3,18 \%$ estavam com SP; $8,17 \%$ com BP e 2,49\% com MBP. Ao comparar os valores de desnutrição obtidos com os valores nacionais, conclui-se que a prevalência de desnutrição na cidade de Serro foi de $10,66 \%$, maior que o valor nacional de 
$2,12 \%$ e maior também que cada região do país: norte $3,4 \%$, nordeste $2,2 \%$, sudeste 1,4 $\%$, sul $2,0 \%$ e centro-oeste $1,6 \%$ (BRASIL, 2006). A ausência de estudos com crianças de 2 a 5 anos anteriores a este, no Município de Serro, não permitiu que se constituísse uma série histórica do estado nutricional de sua população de crianças, mas foi possível comparar os resultados deste estudo com dados de trabalhos de outras localidades e do país. Martins et al. (1995) encontraram 20,5\% de desnutrição entre as 2248 crianças menores de 5 anos na cidade de Niterói, Rio de Janeiro. Monteiro e Conde (2000) coletaram dados de crianças menores de 5 anos na cidade de São Paulo verificando que a frequência de déficits de peso/altura chegou a valores inferiores a 2,5\%. Fernandes et al. (2006) coletaram dados de crianças menores de 5 anos em Mogi-Guaçú, São Paulo e encontraram 5,8\% de subnutrição. No entanto, o valor de desnutrição de 8,6\% encontrado no estudo de Siveira et al. (2010), utilizando uma amostra de 2075 crianças, foi o que mais se aproximou do valor de $10,66 \%$ obtido no presente estudo. Apesar do nosso estudo apresentar $86,14 \%$ de crianças nutridas, é possível observar uma situação séria de desnutrição na cidade do Serro, prevalecendo bastante elevada quando comparada aos valores nacionais, provavelmente relacionada à falta de condições mínimas de existência, como renda insuficiente para a aquisição de alimentos, moradia, serviço, escolaridade, saúde e acesso a informação (LEON-VALENCIA; TERRYBERRO; QUINTANA-JARDINES, 2009). Tais valores podem estar relacionados ao fato de a cidade de Serro localizar-se no Vale do Jequitinhonha-MG, uma das regiões mais pobres do Brasil com o índice de desenvolvimento humano (IDH) de 0,658 e Produto Interno Bruto (PIB) de $\mathrm{R} \$ 4852,25$ (BRASIL, 2004; BRASIL, 2006; BRASIL, 2009).

Apesar dos níveis elevados de desnutrição em todas as idades estudadas, crianças de 5 anos apresentaram o menor índice de desnutrição (8,33\%), no entanto, apresentaram o maior índice de sobrepeso (5\%). Os dados apresentados neste estudo revelam que o índice local de sobrepeso encontrado nessas crianças de 5 anos foi maior quando comparado às demais idades, assim como também quando comparado aos grupos etários em relação a categoria nutricional. Os resultados encontrados de obesidade entre crianças menores de 5 anos na cidade de São Paulo alcançou cerca de $4 \%$ (MONTEIRO; CONDE, 2000). No entando, Fernandes et al. (2006) encontraram 38\% de excesso de peso, em crianças menores de 5 anos em Mogi-Guaçú, São Paulo. Silveira et al. (2010) observaram $11,3 \%$ de obesidade entre as 2075 crianças estudadas. Infelizmente, a obesidade na infância tende a 
continuar na adolescência e na fase adulta, levando ao aumento da morbidade e diminuição da expectativa de vida (ROSSNER, 1998;

ADAMI;

VASCONCELOS, 2008). O excesso de peso pode provocar o surgimento de várias complicações de saúde, como: diabetes melito, hipertensão arterial, dislipidemias, problemas cardíacos e a má formação do esqueleto (ADAMI; VASCONCELOS, 2008). Além disso, os valores de sobrepeso encontrados podem estar relacionados a esta transição no perfil nutricional pelo qual o Brasil está passando, demonstrando uma parcela significativa de crianças com cinco anos de idade com sobrepeso (BRASIL, 2004; BRASIL, 2006; BRASIL, 2009).

É importante ressaltar que entre as 722 crianças estudadas, 66 apresentaram-se entre os percentis 3 e 5, considerado como risco nutricional pela Secretaria de Saúde do Serro, Minas Gerais. Em virtude da inexistência de estudos anteriores que comparam risco nutricional baseado em percentis, não é possível estabelecer a tendência encontrada com os estudos da literatura. No entanto, esse dado é importante, pois essas crianças representam $9,14 \%$ do valor total estudado, logo necessitam acompanhamento adequeado para evitar estágios de desnutrição futura. Para o SISVAN percentis de peso por idade entre 3 e 97 é classificado como nutrido, mas essas 66 crianças encontraram-se próximas do limite inferior da classificação nutrida, estando mais próximas da classificação $\mathrm{BP} /$ desnutrida, logo fazem parte de um grupo de risco nutricional (BRASIL, 2008). Daí a importância da identificação desses grupos, permitindo a realização de programas que melhorem a alimentação infantil evitando a desnutrição protéico-calórica e através de medidas preventivas no intuito de evitar a transição nutrida para BP/desnutrida tornamse, evidentemente, fundamentalis.

Além disso, é de extrema importância que sejam criadas políticas de intervenção nutricional nos grupos de sobrepeso, desnutridos e nos grupos de risco na cidade do Serro, por parte das autoridades locais, para gerar políticas públicas e programas de promoção de saúde com foco diferenciado de acordo com cada faixa etária, previnir a elevação dos índices de desnutrição e obesidade infantil, bem como promover a elevação da taxa de crianças nutridas. Isto pode ser alcançado através de palestras educativas e cartilhas de orientação sobre a importância de uma alimentação infantil balanceada e sobre a prática de atividades físicas regulares. A partir da criação de um programa continuado de vigilância nutricional, perpassando pelas ações básicas de saúde, pode-se esperar que as crianças do Serro, futuramente, atinjam índices mais positivos ou, no mínimo, similares ao restante do Brasil. 


\section{CONCLUSÃO}

Por isso, os resultados deste estudo geram importantes subsídios aos gestores locais em articulação com outros setores e secretarias do município, ao apontar a necessidade de implementação de ações, para favorecer melhorias no contexto marcado pela precária condição de vida dessa população. Daí a importância de estudos transversais como este, pois além de determinar a prevalência, descrevendo uma dada população em um determinado tempo, são fundamentais como primeiro passo para o estabelecimento de medidas preventivas ou terapêuticas para a população estudada (CARDOSO, 2004; BASTOS; DUQUIA, 2007). Posteriormente, novos estudos de caráter multidisciplinar e longitudinal na cidade do Serro são necessários para o melhor entendimento dos processos identificados.

\section{REFERÊNCIAS}

ADAMI, F.; VASCONCELOS, F.A.

Childhood and adolescent obesity and adult mortality: a systematic review of cohort studies. Caderno de Saúde Pública. v.24, Suppl 4, p. 558-568, 2008.

BARROSO, G.S.; SICHIERI, R.; COSTA, R.S. Fatores associados ao déficit nutricional em crianças residentes em uma área de prevalência elevada de insegurança alimentar. Revista Brasileira de Epidemiologia. v.11, p. 484-494, 2008.
BASTOS, J.L.D.; DUQUIA, R.P. Um dos delineamentos mais empregados em epidemiologia: estudo transversal. Scientia Medica. v.17, n.4, p. 229-232, 2007.

BRASIL. Instituto Brasileiro de Geografia e Estatística. Pesquisa de orçamentos familiares 2008-2009: antropometria e estado nutricional de crianças, adolescentes, e adultos no Brasil. Rio de Janeiro: Instituto Brasileiro de Geografia e Estatística. 2009.

BRASIL. Instituto Brasileiro de Geografia e Estatística. Projeção da população do Brasil por sexo e idade para o período 1980-2050. Revisão 2004: Estimativas anuais e mensais da população do Brasil e das unidades da federação e das populações municipais: 19802020. Rio de Janeiro: Instituto Brasileiro de Geografia e Estatística. 2004.

BRASIL. Ministério da Saúde. Protocolos do sistema de vigilância alimentar e nutricional SISVAN. Brasília: Ministério da Saúde. 2008.

BRASIL. Pesquisa Nacional de Demografia e Saúde. Pesquisa Nacional de Demografia e Saúde da Criança e da Mulher. Brasília: Pesquisa Nacional de Demografia e Saúde. 2006.

CARDOSO, S.M. Importância dos Estudos Epidemiológicos. Revista Portuguesa de Cardiologia. v.23, n.5, p. 769-787, 2004.

FERNANDES, I.T.; GALLO, P.R.; ADVINCULA, A.O. Avaliação antropométrica de pré-escolares do município de Mogi-Guaçú, São Paulo: subsídio para políticas públicas de saúde. Revista Brasileira de Saúde Materno Infantil. v.6, p. 217-222, 2006.

FERREIRA-MARIM, M.M.; FABBRO, A.L.D. Estado nutricional avaliado por medidas antropométricas em pré-escolares atendidos pelo Programa de Saúde da Família de Ribeirão Preto-SP. Medicina (Ribeirão Preto). v.45, n.1, p. 23-30, 2012. 
FILHO, M.B.; RISSIN, A. A transição nutricional no Brasil: tendências regionais e temporais. Caderno de Saúde Pública. v.19, Suppl 1, p. 181-191, 2003.

LEON-VALENCIA A.; TERRY-BERRO, B.; QUINTANA-JARDINES, I. Nutrition status in children aged under 5 seen in a medical consulting room of Babahoyo (Republic of Ecuador). Revista Cubana de Higiene Epidemiologia. v.47, n.1, p. 0-0, 2009.

MARINS, V.M.R.V.; COELHO, M.A.S.C.; MATOS, H.J.; AMARAL, N.S.; VALLE, J.; GISMONDI, R.C.; et al. Perfil antropométrico de crianças de 0 a 5 anos do município de Niterói, Rio de Janeiro, Brasil. Caderno de Saúde Pública. v.11, n.2, p. 246253, 1995.

MONTEIRO, C.A.; CONDE, W.L. Tendência secular da desnutrição e obesidade na infância na cidade de São Paulo. Revista de Saúde Pública. v.34, p. 52-61, 2000.

ROSSNER, S. Childhood obesity and adulthood consequences. Acta Paediatrica. v.87, p. 1-5, 1998.

SANTOS, A.L.B.; LEAO, L.S.C.S. Perfil antropométrico de pré-escolares de uma creche em Duque de Caxias, Rio de Janeiro. Revista Paulista de Pediatria. v.26, n.3, p. 218-224, 2008.

SILVA, H.G.V.; CHIARA, V.L.; BARROS, M.E.; REGO, A.L.; FERREIRA, A.; PITASI, B.A.; et al. Diagnóstico do estado nutricional de escolares: comparação entre critério nacional e internacional. Journal of Pediatrics. v.84, n.6, p. 550-555, 2008.

SILVEIRA, K.B.R.; ALVES, J.F.R.; FERREIRA, H.S.; SAWAYA, A.L.; FLORENCIO, T.M.M.T. Associação entre desnutrição em crianças moradoras de favelas, estado nutricional materno e fatores socioambientais. Jornal de Pediatria. v.86, n.3, p. 215-220, 2010.
SPERANDIO, N.; SANT'ANA, L.F.R.; FRANCESCHINI, S.C.C.; PRIORE, S.E. Comparação do estado nutricional infantil com utilização de diferentes curvas de crescimento. Revista de Nutrição. v.24, n.4, p. 565-574, 2011.

STRUFALDI, M.W.L.; PUCCINI, R.F.; PEDROSO, G.C.; SILVA, E.M.K.; SILVA, N.N. Prevalência de desnutrição em crianças residentes no município de Embu, São Paulo, Brasil, 1996-1997. Caderno de Saúde Pública. v.19, n.2, p. 421-428, 2003.

TUMA, R.C.F.B.; COSTA, T.H.M.;

SCHMITZ, B.A.S. Avaliação antropométrica e dietética de pré-escolares em três creches de Brasília, Distrito Federal. Revista Brasileira de Saúde Materno Infantil. v.5, n.4, p. 419428, 2005.

\section{WORLD HEALTH ORGANIZATION.}

Physical status: the use and interpretation of anthropometry indicators of nutritional status. Geneva (Technical Report Series, 854): World Health Organization. 1995. 